\title{
Cell-quintupling: Structural phase transition in a molecular crystal, bis(trans-4- butylcyclohexyl)methanol
}

Kazuya Saito, Takaaki Ikeda, Yasuhisa Yamamura, Hideki Saitoh, Mafumi Hishida, Yutaro Kobayashi, Takeshi Fujita, and Junji Ichikawa

Citation: The Journal of Chemical Physics 146, 074503 (2017); doi: 10.1063/1.4976318

View online: http://dx.doi.org/10.1063/1.4976318

View Table of Contents: http://aip.scitation.org/toc/jcp/146/7

Published by the American Institute of Physics

\section{Articles you may be interested in}

A molecular dynamics investigation of the influence of water structure on ion conduction through a carbon nanotube

The Journal of Chemical Physics 146, 074502074502 (2017); 10.1063/1.4975690

Water dissociation on $\mathrm{Ni}(100), \mathrm{Ni}(110)$, and $\mathrm{Ni}(111)$ surfaces: Reaction path approach to mode selectivity

The Journal of Chemical Physics 146, 074705074705 (2017); 10.1063/1.4976133

General technique for analytical derivatives of post-projected Hartree-Fock

The Journal of Chemical Physics 146, 074104074104 (2017); 10.1063/1.4976145

Island nucleation and growth with anomalous diffusion in one-dimension

The Journal of Chemical Physics 146, 074702074702 (2017); 10.1063/1.4976137

The dissociative chemisorption of $\mathrm{CO} 2$ on $\mathrm{Ni}(100)$ : A quantum dynamics study

The Journal of Chemical Physics 146, 074704074704 (2017); 10.1063/1.4976132

Structure and mechanisms underlying ion transport in ternary polymer electrolytes containing ionic liquids

The Journal of Chemical Physics 146, 074902074902 (2017); 10.1063/1.4976131

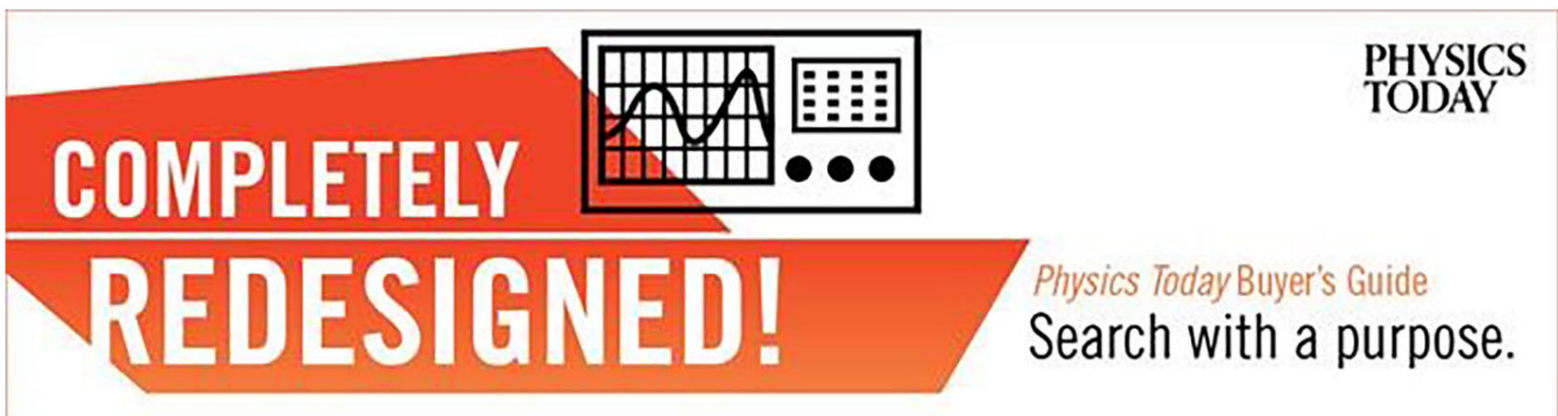




\title{
Cell-quintupling: Structural phase transition in a molecular crystal, bis(trans-4-butylcyclohexyl)methanol
}

\author{
Kazuya Saito, ${ }^{1, a)}$ Takaaki Ikeda, ${ }^{1}$ Yasuhisa Yamamura, ${ }^{1}$ Hideki Saitoh, ${ }^{2}$ Mafumi Hishida, ${ }^{1}$ \\ Yutaro Kobayashi, ${ }^{1}$ Takeshi Fujita, ${ }^{1}$ and Junji Ichikawa ${ }^{1}$ \\ ${ }^{1}$ Department of Chemistry, Faculty of Pure and Applied Sciences, University of Tsukuba, Tsukuba, \\ Ibaraki 305-8571, Japan \\ ${ }^{2}$ Department of Chemistry, Graduate School of Science and Engineering, Saitama University, \\ Saitama 338-8570, Japan
}

(Received 20 December 2016; accepted 31 January 2017; published online 16 February 2017)

\begin{abstract}
A structural phase transition at $151.6 \mathrm{~K}$ of the title compound [bis(trans-4-butylcyclohexyl)methanol] is examined by X-ray diffraction crystallography, Fourier-transform infrared spectroscopy, and adiabatic calorimetry. A general consideration on possible superstructures indicates that a single modulation wave is sufficient to drive this cell-quintupling transition. The entropy of transition determined calorimetrically indicates that two conformations are dominant in the room-temperature phase in contrast to the fivefold disorder expected from the structure of the low-temperature phase. Published by AIP Publishing. [http://dx.doi.org/10.1063/1.4976318]
\end{abstract}

\section{INTRODUCTION}

Phase transitions between crystalline phases having closely related structures are widely involved in the evolution of materials functionality such as ferroelectricity. ${ }^{1-3}$ Such phase transitions offer the stage for detailed discussions in the context of the group theory first proposed by Landau ${ }^{4}$ (Landau's thermodynamic phenomenology). Landau's phenomenology puts forward the criteria that govern the possible occurrence of a continuous transition. A prominent example includes the discontinuous (first-order) nature of the phase transition between isotropic liquid and nematic liquid crys$\mathrm{tal}^{5}$ though it is not a transition between crystalline phases. The criterion that works in this example is called the Landau condition: ${ }^{4,6}$ the thermodynamic potential relevant for a continuous transition must not have the third-order term in terms of the order parameter. In the above example, due to the asymmetry between positive and negative nematic order parameters, there is no reason to vanish for the third-order term, resulting in the first-order transition. ${ }^{5}$

The appearance of superstructures is widely observed for transitions between crystalline phases. Landau's phenomenology allows a continuous transition for limited cases (such as the cell-doubling in a single lattice axis) but does not for others. ${ }^{4,6,7}$ This is, first of all, because the wavevector of the structural modulation driving the phase transition is on the boundary of the (first) Brillouin zone, known as the Lifshitz condition. The condition, however, may be violated in such a case that a phase transition is to a "superstructure" having an incommensurate period with respect to the mother lattice. ${ }^{2,8}$ Besides, one may count, as a cause of the impossibility of the higher-order integer superstructure, the factor that plural modulation waves are necessary to establish complex superstructures. The participation of plural order parameters generally suppresses the

a)Electronic mail: kazuya@chem.tsukuba.ac.jp possibility of the occurrence itself of such phase transitions. Reported and discussed in this paper is, however, such a phase transition with cell-quintupling in a molecular crystal.

The compound subjected to the present study is bis(trans4-butylcyclohexyl)methanol (abbreviated as BBCHM hereafter), the chemical structure of which is shown in Fig. 1 with $\mathrm{R}=\mathrm{C}_{4} \mathrm{H}_{9}$. The study originally started to characterize its (possible) glass transition because its mother compound, dicyclohexylmethanol (DCHM, R = H in Fig. 1), exhibits a glass transition of the isotropic liquid consisting of globular tetramers, ${ }^{9}$ which results from the severe steric hindrance at the molecular level. ${ }^{10,11}$ Basic characterization of newly synthesized BBCHM revealed that the crystal undergoes a peculiar structural phase transition with cell-quintupling. This paper reports the results of Fourier-transform infrared spectroscopy (FT-IR) at various temperatures, adiabatic calorimetry below $350 \mathrm{~K}$, and structure solutions at room-temperature (RT) and low-temperature (LT). Through a general consideration of possible superstructures, the character of the phase transition of BBCHM that is discussed is that a single modulation wave is sufficient to drive this cell-quintupling transition.

\section{EXPERIMENTAL}

\section{A. Synthesis}

BBCHM was synthesized as summarized in Fig. 2. 4-chlorocyclohexan-1-one was prepared according to the literature procedure by the ring-opening of 7-oxabicyclo[2.2.1] heptane and subsequent oxidation. ${ }^{12}$ Butyl group was installed on the cyclohexane ring by a Wittig/hydrogenation sequence. The resulting cis/trans mixture of 1-butyl-4chlorocyclohexane was converted to the corresponding Grignard reagent, which was in turn treated with $1,1^{\prime}$ carbonyldiimidazole to afford a sole isomer of bis(4butylcyclohexyl)methanone. Finally, an isomer of bis(4butylcyclohexyl)methanol was obtained via reduction with 


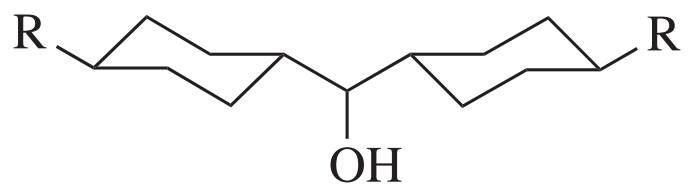

FIG. 1. Molecular structure of analogues of DCHM. $\mathrm{R}=\mathrm{H}$, dicyclohexylmethanol (DCHM); $\mathrm{R}=\mathrm{C}_{4} \mathrm{H}_{9}$, bis(trans-4-butylcyclohexyl)methanol (BBCHM). Hydrogen atoms tetrahedrally bonding to carbon atoms are omitted for clarity.

$\mathrm{NaBH}_{4}$. The X-ray crystallography on single crystals revealed that the final compound is the (trans, trans) isomer (BBCHM). Recrystallization from the chloroform solution resulted in crystalline BBCHM. Some details and spectroscopic data of relevant compounds are given in the supplementary material.

\section{B. Measurements}

\section{FT-IR}

Powdered specimen was sandwiched between $\mathrm{KBr}$ disks under reduced pressure, and then exerted pressure to form a pellet. Spectra were recorded using JASCO FT/IR-550 between 80 and $360 \mathrm{~K}$.

\section{Calorimetry}

As-synthesized specimen was dried under vacuum for 2 nights. The sample crushed gently was loaded into a calorimeter vessel (gold-plated copper) under a helium atmosphere, and sealed with a lead gasket. The vessel was equipped with a platinum resistance thermometer (MINCO S1059), the temperature scale of which is based upon the ITS-90. The vessel was set in a laboratory-made adiabatic calorimeter, which basically shares the construction and measuring system as those described in the literature. ${ }^{13}$

The mass of the sample loaded into the calorimeter vessel was $0.44943 \mathrm{~g}(1.45675 \mathrm{mmol})$ after the buoyancy correction. Measurements were done in the so-called intermittent heating mode. The temperature increment for each data point was about $1 \%$ of the temperature outside the vicinity of each phase transition. Thermal equilibrium within the vessel was attained within 3 min below $20 \mathrm{~K}$, and $10 \mathrm{~min}$ above $100 \mathrm{~K}$, irrespective of anomaly in heat capacity. The sample contributed to the total heat capacity including those of the vessel, gasket, and helium gas by $10 \%$ at $300 \mathrm{~K}$. Heat capacities of the sample were obtained after subtracting, from the raw experimental heat capacities, those of the vessel and others determined based on separate measurements. Due to a relatively small amount of the sample used for the measurement in comparison with those in usual measurement using this calorimeter (ca. $1 \mathrm{~g}$ or more), the scatter of heat capacity data of the sample was rather large.

\section{X-ray crystallography}

Single crystals were picked up from the recrystallized specimen from chloroform in the final stage of the synthesis. The conditions for data collection are given in the supplementary material. It is noted that the diffractometer (Bruker APEX-II CCD) is equipped with a CCD camera, which enables us to observe the appearance/absence of possible superlattice reflections. Data reduction and cell refinement were performed by SAINT. ${ }^{14}$ Structures were solved by the direct method using SHELXS-97, ${ }^{15}$ and refined using SHELXL-2014. ${ }^{16}$ The crystal data for the room- and low-temperature phases are summarized in the supplementary material. Crystallographic data have been deposited with Cambridge Crystallographic Data Centre. ${ }^{17}$

\section{RESULTS}

\section{A. FT-IR}

The preliminary experiments using differential scanning calorimetry indicated that the as-synthesized sample exhibits a small thermal anomaly just below the melting and that the sample crystallized from the melt does not. FT-IR spectra were thus recorded in separate runs starting from the room temperature in cooling and heating. A part of spectrum at room temperature is shown in the inset of Fig. 3. It is known the $\mathrm{OH}$ group involved in $\mathrm{H}$-bond gives a rather broad absorption around $3400 \mathrm{~cm}^{-1}$ whereas free $\mathrm{OH}$ does around $3600 \mathrm{~cm}^{-1} .{ }^{18}$ The absence of a band around $3600 \mathrm{~cm}^{-1}$ implies that all $\mathrm{OH}$ groups are involved in $\mathrm{H}$-bonds in solid BBCHM, as in the mother compound DCHM. ${ }^{10,11}$

To ascertain the complete formation of H-bonds and its rupture on the course of melting, the relative intensity of the $\mathrm{H}$ bonded $\mathrm{OH}$ band is plotted in Fig. 3. The intensity (integrated intensity) was obtained as follows: (i) the area involved in the $\mathrm{OH}$ band is normalized against that of the $\mathrm{CH}$ stretching band (2800-3050 $\left.\mathrm{cm}^{-1}\right)$ assuming a smooth background for

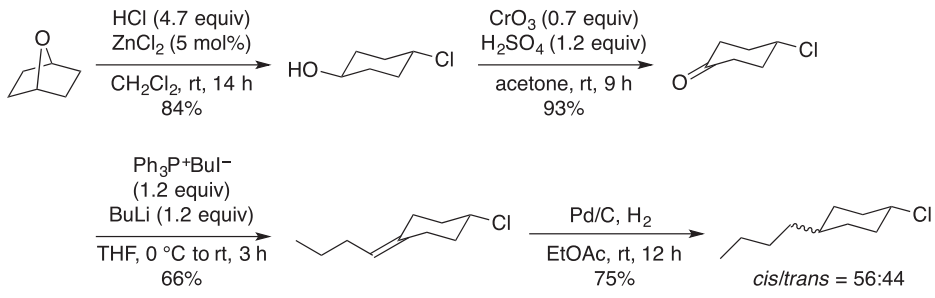
1) $\mathrm{Mg}(1.1$ equiv)
$\mathrm{THF}$, reflux, $1.5 \mathrm{~h}$
2) Carbonyldiimidazole
$\quad(0.5$ equiv $)$ THF, $-78^{\circ} \mathrm{C}$ to $\mathrm{rt}, 25 \mathrm{~h}$ $44 \%$
FIG. 2. Synthetic route of BBCHM. Percentage indicates the yield of the compound based on the amount of a preceding compound shown at the beginning of a respective arrow. 


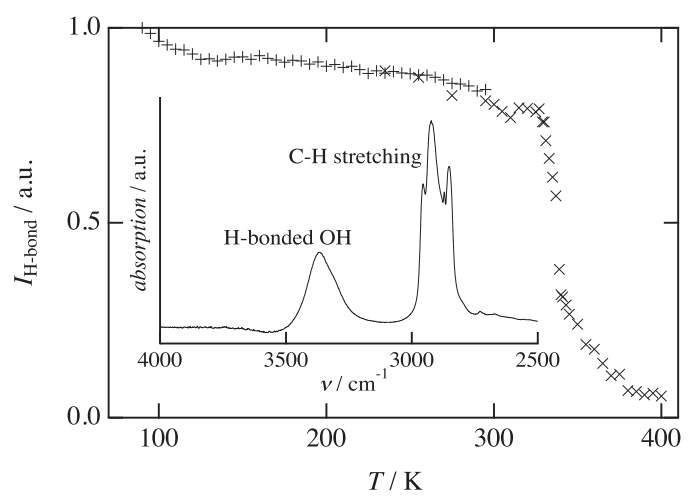

FIG. 3. Temperature dependence of the intensity of the IR band arising from the H-bonded $\mathrm{OH}$ in solid $(<330 \mathrm{~K})$ and liquid $(>330 \mathrm{~K})$ of BBCHM. Different symbols represent data in separate measurement runs from room temperature on heating and cooling. Inset shows the IR spectrum at room temperature in the $\mathrm{CH}$ and $\mathrm{OH}$ stretching range.

each spectrum and (ii) the normalized intensity is matched at $295 \mathrm{~K}$ for two runs. The abrupt decrease around $330 \mathrm{~K}$ corresponds to the fusion of tiny solids. It is interesting to note that the decrease in the intensity is more moderate in the liquid state. This resembles the case in DCHM. ${ }^{11}$

Temperature dependence of the band shape in the $\mathrm{CH}$ stretching region is shown in Fig. 4 except for the band arising from it of the central carbon atom, which splits into two weak bands with maximums around 2730 and $2670 \mathrm{~cm}^{-1}$ because of the asymmetry of two butyl groups around the (pseudo-) molecular twofold axis (the bisector of the angle $\angle \mathrm{OCO}$ as explained later). Although the band shape gradually changes upon cooling, three distinct bands can be recognized only at low temperatures (below ca. $150 \mathrm{~K}$ ). They imply a possible existence of a phase transition. A comparison of the band shape

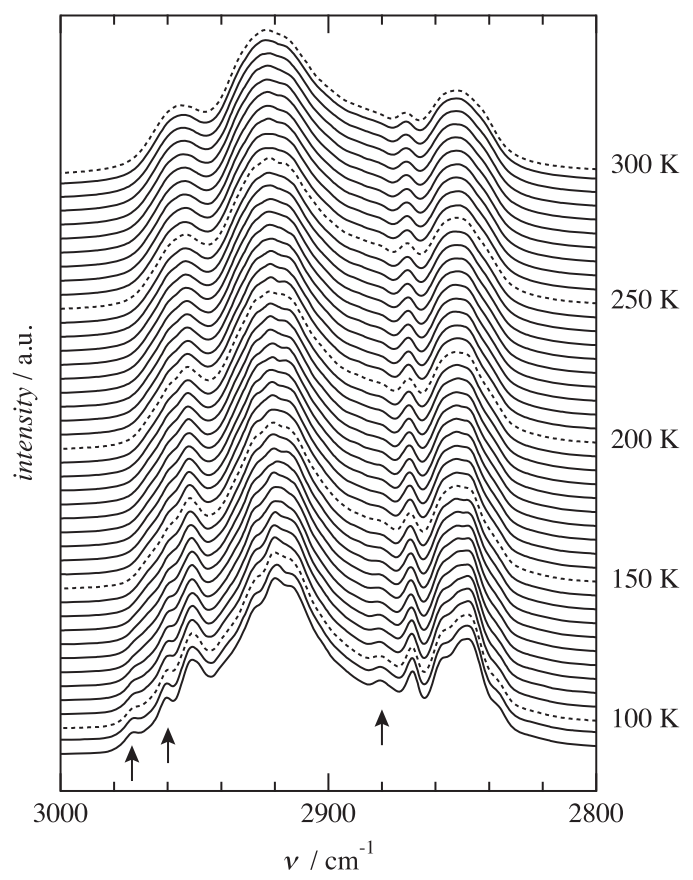

FIG. 4. IR spectra (at interval of $5 \mathrm{~K}$ ) in the $\mathrm{CH}$ stretching region of BBCHM below room temperature. Temperatures of spectra shown by broken curves are indicated. Arrows indicate the bands appearing below ca. $150 \mathrm{~K}$. with that of DCHM ${ }^{11}$ (without butyl groups) clearly indicates that the three bands are from butyl groups.

\section{B. Adiabatic calorimetry}

The irreversibility of solid phases was confirmed through the difference in heat capacity: The data on the solid recrystallized from melt differed from those of the as-synthesized sample. The following description is based on the data that have not experienced the liquid state except the heat capacities of normal and supercooled liquids.

Experimental heat capacities of the as-synthesized sample are shown in Fig. 5 for the whole temperature range studied. Since separate examinations by differential scanning calorimetry indicate that the melting temperature is about $330 \mathrm{~K}$, the sample is in the crystalline state below this temperature. A sharp anomaly and a step-like anomaly are discernible around $152 \mathrm{~K}$ and $245 \mathrm{~K}$, respectively, in addition to anomalies around $330 \mathrm{~K}$.

The anomaly around $152 \mathrm{~K}$ is attributed to a phase transition since its temperature reasonably matches the expected one based on the FT-IR results (Fig. 4). To see the detail of the shape of this anomaly, an enlarged plot is shown in Fig. 6. Although the anomaly accompanies tails on the low-temperature side, this phase transition is definitely of first order, as evidenced by supercooling of the high-temperature phase indicated by crosses and plus signs. The transition temperature is determined as $(151.6 \pm 0.1) \mathrm{K}$. Assuming a smooth interpolating curve as the baseline, the excess heat capacities were separated and integrated. The enthalpy and entropy of transition including the contributions of tails are determined to be $(1.14 \pm 0.01)$ $\mathrm{kJ} \mathrm{mol}^{-1}$ and $(7.59 \pm 0.03) \mathrm{J} \mathrm{K}^{-1} \mathrm{~mol}^{-1}$, respectively. The latter is larger than a typical magnitude expected for a simple order-disorder transition $\left(R \ln 2 \approx 5.8 \mathrm{~J} \mathrm{~K}^{-1} \mathrm{~mol}^{-1}\right)$.

Although the anomaly around $230 \mathrm{~K}$ resembles that involved in a glass transition with respect to a step-like

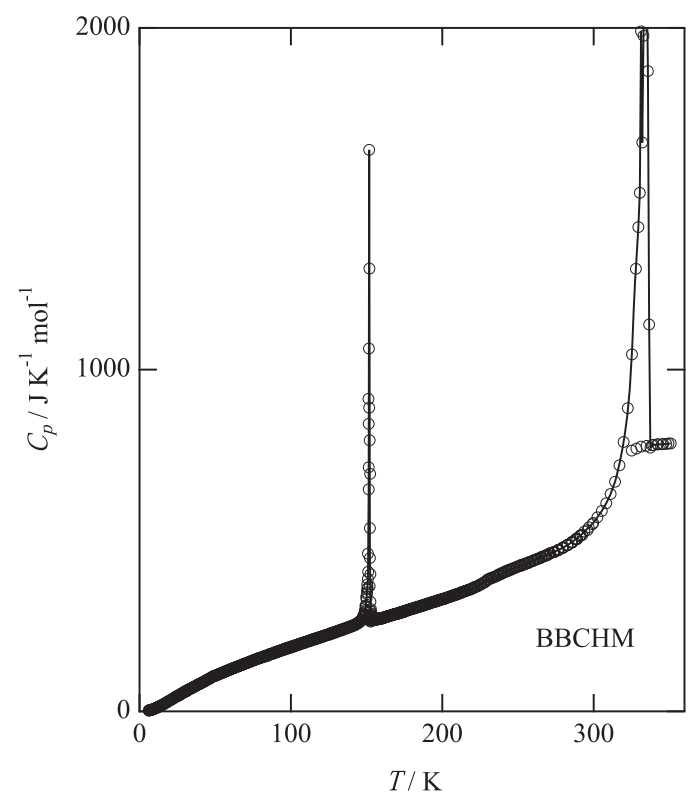

FIG. 5. Experimental heat capacities of BBCHM. The data points smoothly continuous to those above $330 \mathrm{~K}$ are of the supercooled liquid. 


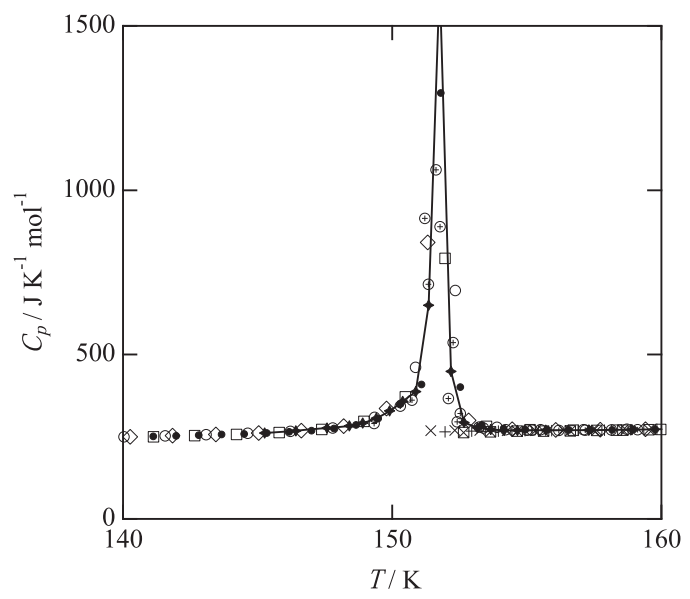

FIG. 6. Experimental heat capacities of BBCHM around $150 \mathrm{~K}$. Different symbols represent data in separate measurement runs.

increase, it does not accompany a behavior typical for a glass transition (enthalpy relaxation) in temperature drift during the equilibration periods. No anomalous behavior was also encountered in FT-IR. The origin of this anomaly is unknown at present.

A seeming irreversibility of the crystal growth, i.e., different crystalline phases between the as-synthesized and meltgrown samples, prevented the reliable investigation of the melting behavior. However, it is noted that the crystallization from the melt proceeded on cooling more easily than DCHM,,${ }^{9,11}$ resulting in another phase from the as-synthesized sample characterized thoroughly in this study.

\section{Naïve structure analysis using X-ray}

The FT-IR spectrum of the powdered specimen (even at room temperature) suggests that all hydroxy groups participate in H-bonds. Although the following analysis of the crystal structure is apparently incompatible with this, we start with a series of naïve analyses of crystal structure in this section. A plausible solution will be given later with some discussions.

\section{Room-temperature phase}

BBCHM crystallizes into a monoclinic system at room temperature as summarized in Table I. The asymmetric unit of the structure contains a single molecule. The structure was successfully refined by assuming disorders in the direction of $\mathrm{C}-\mathrm{OH}$ (up and down equally with respect to the $\boldsymbol{b}$-axis) and the two conformations of a butyl moiety. Because of the similarity in molecular orientations and structures, the cell corresponding

TABLE I. Lattice parameters of room-temperature (RT) and low-temperature (LT) phases of BBCHM.

\begin{tabular}{lcc}
\hline \hline & RT phase $(295 \mathrm{~K})$ & LT phase $(100 \mathrm{~K})$ \\
\hline Crystal system & Monoclinic & Monoclinic \\
Space group & $P 2_{1} / n$ & $P 2_{1} / n$ \\
$a / \AA$ & $18.9126(12)$ & $44.559(3)$ \\
$b / \AA$ & $5.1435(3)$ & $5.0827(3)$ \\
$c / \AA$ & $22.1444(12)$ & $45.694(3)$ \\
$\beta /$ deg & $95.480(4)$ & $101.0569(14)$ \\
$Z$ & 4 & 20 \\
\hline \hline
\end{tabular}

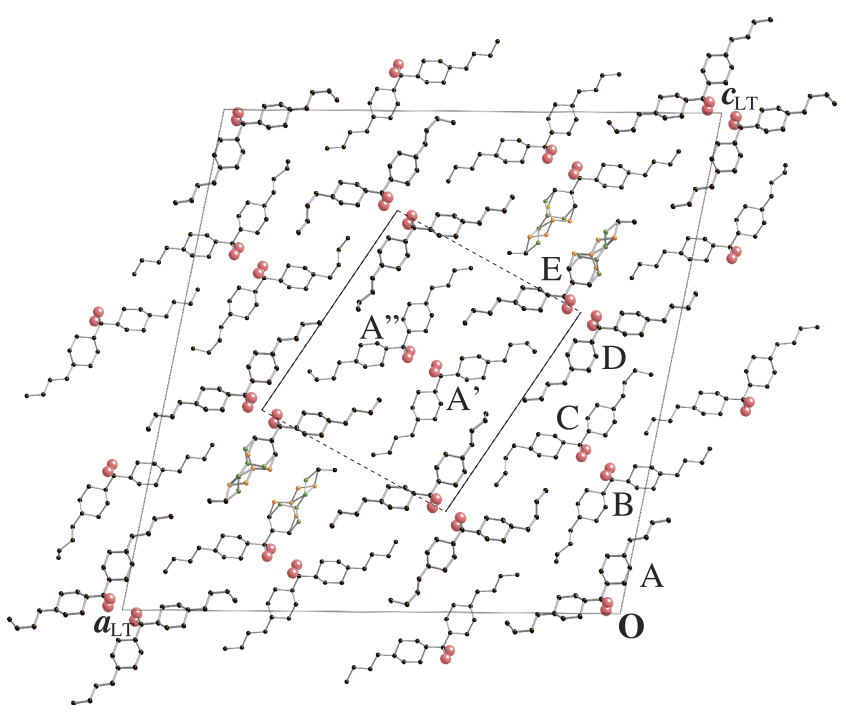

FIG. 7. Crystal structure of BBCHM in the LT phase viewed along the $\boldsymbol{b}$ axis. Hydrogen atoms are omitted for clarity. Molecules A-E are independent molecules. Molecules $\mathrm{A}^{\prime}$ and $\mathrm{A}^{\prime \prime}$ are related to each other by an inversion, and related to the molecule A by respective symmetries. The unit cell of the RT phase is shown by dotted lines.

to the RT phase drawn in that of the LT phase (shown in Fig. 7) well expresses the structure of the RT phase except for the disorder of a butyl moiety of all molecules in the RT phase. The mode of disorder is similar to that found for molecule $\mathrm{E}$ (one out of five independent ones) of the LT phase as shown in Fig. 8. Ignoring the disorder of the butyl moiety, the bisector of the angle $\angle \mathrm{OCO}$ of each molecule can be regarded as a pseudo-twofold axis.

The structure is rather simple. Indeed, the space group is well suited for simple molecular crystals as pointed out by Kitaigorodsky ${ }^{19}$ and is the most frequently observed one. ${ }^{20}$ On the other hand, a short $b(=|\boldsymbol{b}|)$ indicates that only a single molecule is contained in a cell along this direction. This is problematic for constructing $\mathrm{H}$-bonds in a just enough way. The distance between the lower $\mathrm{O}$ atoms belonging to a pair of molecules related by an inversion (like molecules $\mathrm{A}^{\prime}$ and $\mathrm{A}^{\prime \prime}$ in Fig. 7) is 2.743(6) $\AA$, while that between the upper O atom of the original molecule and the lower $\mathrm{O}$ atom belonging

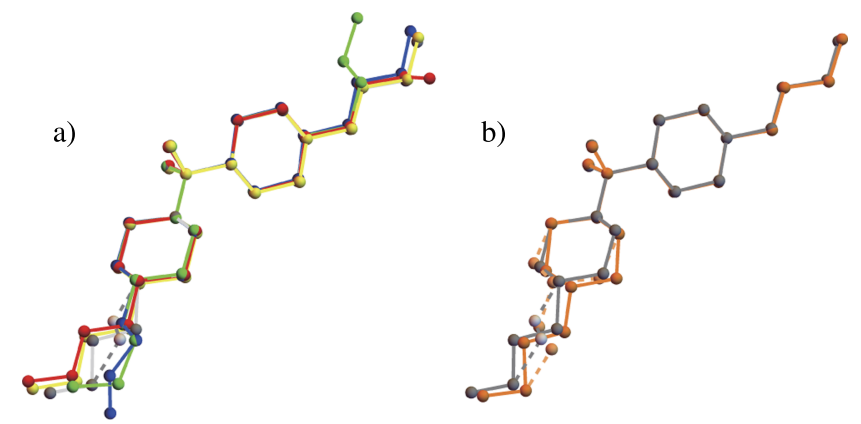

FIG. 8. Comparison of molecular structures in the RT and LT phases of BBCHM. (a) Molecules in the RT phase (gray) and A (red), B (blue), C (green), and D (yellow) in the LT phase; (b) molecules in the RT phase (gray) and $\mathrm{E}$ (orange) in the LT phase. Two oxygen atoms appear due to the structural disorder as described in the text. Minor conformers of butyl groups are shown in lighter color with broken lines as bonds for the molecule of the RT phase and the molecule $\mathrm{E}$ of the LT phase. Hydrogen atoms are omitted for clarity. Radius of spheres (atoms) is $0.2 \AA$. 
to a molecule shifted by $\boldsymbol{b}_{\mathrm{RT}}$ is 2.750 (7) $\AA$. These are close to O-O distances (2.76(1) $\AA$ and 2.77(1) $\AA$ ) in the crystal of the mother compound, DCHM, which forms a closed tetramer via cyclic H-bonds. ${ }^{10}$ This suggests the formation of tetramers in the crystal of BBCHM.

\section{Low-temperature phase}

The low-temperature phase has a large unit cell with the same space group having the short $b$, in comparison with the room-temperature phase in Table I. The central DCHM moieties remain essentially in the same arrangement as that in the RT phase with the disorder concerning the direction of the C$\mathrm{OH}$. The crystal structure viewed along the $b$-axis is shown in Fig. 7. The relations between reciprocal lattice vectors of RT and LT phases are expressed as

$$
\begin{aligned}
& \boldsymbol{a}_{\mathrm{LT}}^{*}=\left(2 \boldsymbol{a}_{\mathrm{RT}}^{*}-\boldsymbol{c}_{\mathrm{RT}}^{*}\right) / 5, \\
& \boldsymbol{b}_{\mathrm{LT}}^{*}=\boldsymbol{b}_{\mathrm{RT}}^{*}, \\
& \boldsymbol{c}_{\mathrm{LT}}^{*}=\left(\boldsymbol{a}_{\mathrm{RT}}^{*}+2 \boldsymbol{c}_{\mathrm{RT}}^{*}\right) / 5 .
\end{aligned}
$$

The relation on the $\boldsymbol{a}_{\mathrm{LT}}^{*} \boldsymbol{c}_{\mathrm{LT}}^{*}$-plane is illustrated in Fig. 9. The same relations as Eq. (1) apply among the lattice vectors as easily verified in Fig. 7 if asterisks indicating reciprocal vectors are omitted.

The comparison of molecular structures are shown in Fig. 8, where the radius of spheres (atoms) is $0.2 \AA$. The central DCHM part of the molecules A-D has essentially the same structure as that of molecules in the RT phase whereas the conformation of butyl groups is largely different. On the other hand, the molecule $\mathrm{E}$ has a different structure also in a half unit of DCHM part from molecules in the RT phase. It is however interesting to note that the molecule $\mathrm{E}$, together with the molecule $\mathrm{D}$, is rather similar in structure to molecules in the RT phase if the conformation of butyl groups is taken into account.

Pairs of molecules can be identified as in the RT phase because of the close similarity of the structures. While the

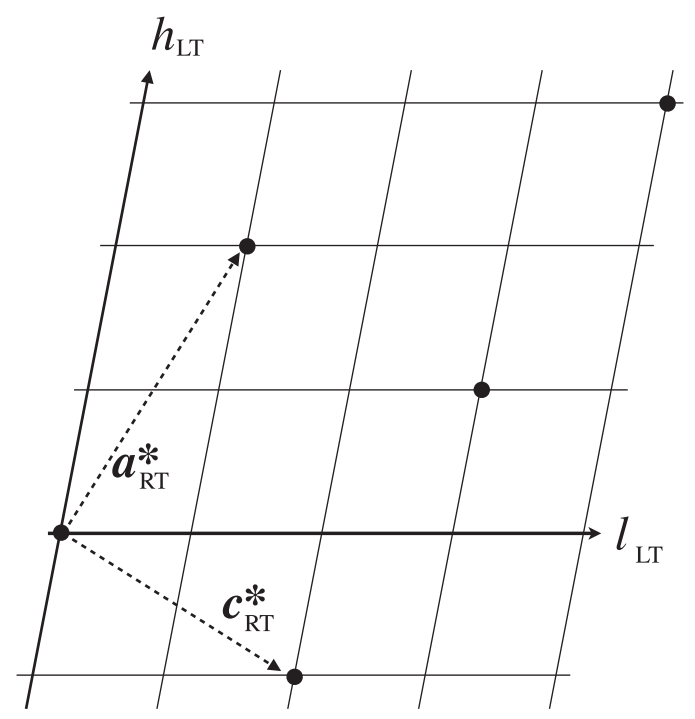

FIG. 9. Relation between the reciprocal lattice vectors between the roomtemperature (RT) and low-temperature (LT) phases of BBCHM on the $\boldsymbol{a}_{\mathrm{LT}}^{*} c_{\mathrm{LT}^{*}}^{*}$ plane. The lattice corresponds to the LT phase. The reciprocal lattice points of the RT phase are indicated by filled circles. molecules $\mathrm{A}^{\prime}$ and $\mathrm{A}^{\prime \prime}$ in pair $\mathrm{A}^{\prime} \mathrm{A}^{\prime \prime}$ in Fig. 7 are related by an inversion, those in each pair of $\mathrm{BC}$ and $\mathrm{DE}$ are crystallographically independent to each other. The structural changes upon the phase transition are roughly summarized as follows: The butyl groups changes their conformation in pairs of $\mathrm{A}^{\prime} \mathrm{A}^{\prime \prime}$ and $\mathrm{BC}$ while the change is small in the DE pair.

The $\mathrm{O}-\mathrm{O}$ distances range in $2.709-2.730 \AA$ (average: $2.721(7) \AA$ ) nearly within the $\boldsymbol{a}_{\mathrm{LT}} \boldsymbol{c}_{\mathrm{LT}}$-plane and 2.719 $-2.743 \AA(2.730(8) \AA)$ along the $\boldsymbol{b}_{\mathrm{LT}}$-axis. These are comparable to those in DCHM, although these are slightly shorter than those in the RT phase with longer ones along the $\boldsymbol{b}_{\mathrm{LT}}$-axis. The detailed comparison and discussion on structures such as the large number of independent molecules ${ }^{21}$ are left for a future work.

\section{DISCUSSION}

\section{A. H-bonds and crystal structures}

The complete formation of H-bonds implied by FT-IR results and the short $b$-axis severely conflict with each other. Only way to form H-bonds completely is to assume that the apparent unit cell indicated in Table I is a half of the true period along the $\boldsymbol{b}$-axis. As described in the structural results, a closed $\mathrm{H}$-bond tetramer consisting of a molecular pair in a cell and another pair in the adjacent cell can be constructed within the structure of the RT phase. Because of the pseudotwofold symmetry of the central moiety of each molecule, this construction of $\mathrm{H}$-bond tetramers naturally rationalizes the equal probability of the up and down orientations of the $\mathrm{C}-\mathrm{OH}$ bonds. Since two adjacent pairs are necessary to form a tetramer, the period along the $\boldsymbol{b}$-axis is, at least, to be doubled with respect $b$. Although there is a choice of phases of putting H-bond tetramers along the $\boldsymbol{b}$-axis for one at corners of the cell and the other at centers, two choices result in the same structure belonging to the space group $P c$. The situation is quite similar for the LT phase assuming that each stack along the $\boldsymbol{b}$-axis retains the phase of the stack in the RT phase because molecules are too long to achieve the flip around the pseudotwofold axis necessary for the flip of the direction of the $\mathrm{C}-\mathrm{OH}$ bond.

Experimentally, no superlattice reflection was identified along the $\boldsymbol{b}^{*}$-axis. This implies that the perfect order with the doubled periodicity does not occur. The fragility of the longrange order in one dimension ${ }^{4}$ probably destroys the order even within a stack along the $\boldsymbol{b}$-axis. Besides, the presence of the choice of the phase for putting "ordered" stacks is to be considered. It is thus suggested as a plausible scenario that each stack along the $\boldsymbol{b}$-axis is organized well enough to diminish almost completely the IR band arising from non-H-bonded $\mathrm{OH}$ groups but relative phases of stacks are essentially random. This guarantees the seeming disorder of the directions of $\mathrm{C}-\mathrm{OH}$ bonds with the population 1:1.

Is this structure consistent with the condition of crystal growth? The answer is positive. In the case of DCHM, ${ }^{9,11}$ the steric repulsion and strong tendency for H-bond result in the formation of closed tetramers. These seem to apply to the case of BBCHM, and bring about the crystallization of tetramers. The incompleteness of tetramer formation due to finite temperatures causes the crystallization to the "disordered" 
crystal having $P 2_{1} / n$ symmetry, which is recovered by disorders.

\section{B. General consideration on superstructures}

There are many crystals that undergo a structural phase transition into a larger unit cell than the original one upon cooling. Now, the ratio of $Z$, the number of molecules within a unit cell, is termed multiplicity $m$. Within the context of Landau theory, a continuous phase transition (of the second kind) with $m=1,2$ may be expected starting from the space group $P 2{ }_{1} / n$ of the RT phase of BBCHM (or equivalently $P 2_{1} / c$ and $P 2_{1} / a$, depending on the choice of lattice vectors with the unique $b$-axis). ${ }^{7}$ The phase transition in BBCHM is of $m=5$. However, the relation between the reciprocal lattices between the RT and LT phases clearly indicates that a structural modulation commensurate with the RT structure may drive the phase transition between them. In this section, through a general consideration on possible superstructures, considered is to what extent the $m=5$ transition is reasonable.

Figure 9 shows that there are four new reciprocal lattice points (of the LT phase) within a unit reciprocal lattice of the RT phase. They are grouped into two: $\left\{c_{\mathrm{LT}}^{*}, a_{\mathrm{RT}}^{*}+c_{\mathrm{RT}}^{*}-c_{\mathrm{LT}}^{*}\right\}$ and $\left\{\boldsymbol{c}_{\mathrm{RT}}^{*}+\boldsymbol{a}_{\mathrm{LT}}^{*}, \boldsymbol{a}_{\mathrm{RT}}^{*}-\boldsymbol{a}_{\mathrm{LT}}^{*}\right\}$ on the basis of the displacement from lattice points of the mother lattice of the RT phase. Namely, the former group can be reachable by $\pm \boldsymbol{c}_{\mathrm{LT}}^{*}$ whereas the latter by $\pm a_{\mathrm{LT}}^{*}$. Although the possibility of grouping seemingly requires modulations in two directions, the two groups are related as harmonics because, e.g., $\boldsymbol{c}_{\mathrm{RT}}^{*}+\boldsymbol{a}_{\mathrm{LT}}^{*}=2 \boldsymbol{c}_{\mathrm{LT}}^{*}$, clearly indicating that a member of the latter group is a double of a member of the former. This means that a single modulation wave can drive the transformation from the RT phase to the LT phase if the modulation wave contains the second harmonics. Such a coverage of reciprocal lattice points is generally impossible, for example, in cases of $\boldsymbol{a}_{2}^{*}=2 \boldsymbol{a}_{1}^{*}$ and $\boldsymbol{b}_{2}^{*}=2 \boldsymbol{b}_{1}^{*}$ with $m=4$, and $\boldsymbol{a}_{10}^{*}=\left(3 \boldsymbol{a}_{1}^{*}-\boldsymbol{c}_{1}^{*}\right) / 10$ and $\boldsymbol{c}_{10}^{*}=\left(\boldsymbol{a}_{1}^{*}+3 \boldsymbol{c}_{1}^{*}\right) / 10$ with $m=10$, exemplified in Fig. 10. Both need two modulation waves (containing harmonics) to cover completely the new reciprocal lattice points. Note that the latter example is oblique with respect to the mother lattice as in the case of Eq. (1).
There exist lattice points where a single modulation wave and its harmonics cannot reach: In the case of $m=10,\left(h_{L T}\right.$, $\left.l_{L T}\right)=(0, l)$ and $(2, l)$ are reachable from the lattice points (of the mother phase) by steps of multiples of $(0,1)$ but $(1, l)$ unreachable.

Let us examine $m$ that allows the complete coverage of new reciprocal lattice points by a single modulation wave and harmonics, while referring to Fig. 10. We restrict ourselves to the consideration in two-dimension because two independent vectors, at least, are necessary in three-dimension. The number of new reciprocal lattice points is $(m-1)$ for a phase transition with $m$. The case $m=1$ is a well-known ferroic phase transition, and a single lattice point emerges for $m=2$. In these two cases, all reciprocal points are trivially covered by a single modulation wave. In the case of $m=3$, two points newly appear symmetrically around the lattice point of the mother system. They are harmonics to each other. The case of $m=4$ has two ways of transition: three points in line or one at the center and two at respective midpoints as in Fig. 10. The former is trivially covered by harmonics while the latter definitely requires two modulation waves. In the case $m=5$, only two ways are possible concerning the arrangement of new lattice points as in $m=4$. Namely, four points inline or the pattern observed in BBCHM (Fig. 9).

We now summarize the above findings in a general form. It is noted that new points must be arranged just enough inside a parallelogram or on its sides. This limits ways of arrangements of new lattice points. Superstructures appearing upon a phase transition is divided into two groups: simple $(i \times j)$-superstructure $(i, j \in \mathbb{N})$ and oblique one. The former includes, as special cases, simple $(1 \times j)$-superstructures, for which the $(m-1)$ new reciprocal lattice points are trivially covered by a single modulation wave and its harmonics. On the other hand, simple $(i \times j)$-superstructures $(i, j \geq 2)$ definitely require two (independent) modulation waves and their harmonics. Oblique superstructures correspond to $\left(i^{*} \times j^{*}\right)$-arrangements $\left(i^{*}, j^{*} \in \mathbb{N}\right.$, and $\left.i^{*}, j^{*} \geq 2\right)$ of new reciprocal lattice points such as those shown in Fig. 9 and the $m=10$ case in Fig. 10. The new reciprocal lattice is completely covered by a single modulation wave and its harmonics in, say, the $j^{*}$ direction for the $\left(2 \times j^{*}\right)$ arrangement,

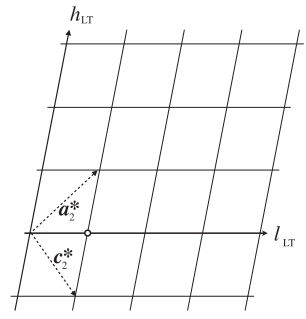

$m=2$

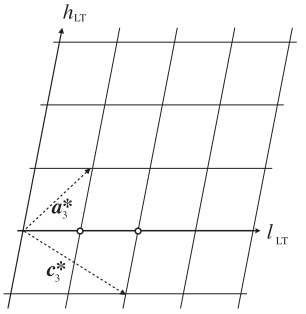

$m=3$
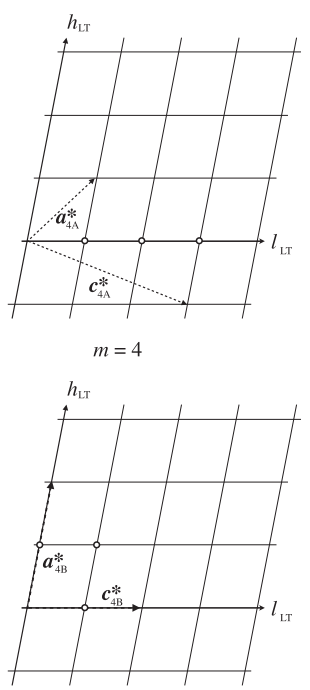

FIG. 10. Examples of arrangements of new reciprocal lattice points for $m=2$, 3,4 , and 10. For ease of illustration and comparison with Fig. 9, reciprocal lattices of superstructures (with a lower symmetry) are kept the same, in contrast to real cases where the unit cell of the high-symmetry phase is expected to remain the same. New reciprocal lattice points emerging upon the phase transition to the superstructure are indicated by open circles.

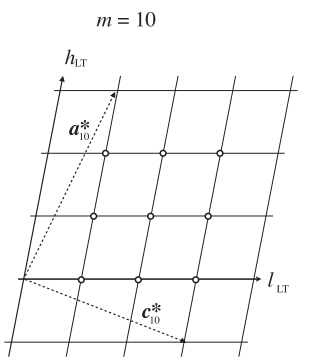


whereas the complete coverage is impossible for $\left(i^{*} \times j^{*}\right)$ -arrangements $\left(i^{*}, j^{*} \geq 3\right)$ as observed in the $m=10$ case in Fig.10.

The above consideration solely relies on the translational symmetry of crystalline lattice. Any plane in the reciprocal space may be considered. However, occurrence of phase transitions with a large $m$ would depend on specific situation of respective crystals. In BBCHM, for example, the arrangements of molecules (according to the space group) seem important. In this respect, Landau's spirit that the symmetry governs phase transitions could work as a guiding principle, though Landau's phenomenology of phase transition does not contribute in most cases. Full exploration of space groups in view of the present consideration is beyond the scope of this paper and left for future.

\section{Phase transition in BBCHM}

Within the context developed in Section IV B, the experimentally observed $(2 \times 2)$-arrangement of new reciprocal lattice points for BBCHM is the minimum case that is not inline (the trivial coverage) but completely covered by a single modulation wave with harmonics. If the crystal consists of large discrete molecules such as BBCHM, it is implausible that the modulation wave is purely sinusoidal. In this sense, the superstructure related by Eq. (1) has a special meaning. In the case of BBCHM, the factor that stabilizes the $m=5$ structure would be symmetrical: The transition keeps a rather high symmetry.

The necessary wavevector of the modulation wave to drive the phase transition in BBCHM locates on the $a_{\mathrm{RT}}^{*} c_{\mathrm{RT}}^{*}$-plane with its end at a general point in the reciprocal space. The wavevector is not "active" in terms of the Landau theory, ${ }^{4,6}$ accordingly. This basically explains the first-order nature of the phase transition. The requirement for second harmonic in the modulation wave has cooperative effects: The presence of plural order parameters generally prefers a discontinuous transition.

Note that the orientational disorder of the $\mathrm{C}-\mathrm{OH}$ groups is plausibly assumed to be quenched because of the large stabilization by $\mathrm{H}$-bonds and the bulkiness of molecules as discussed in relation to crystal structures. It is, at this point, noteworthy that the phase transition is quite sharp even though it occurs in a severely disordered system.

The maximum of the entropy increment is $R \ln m$ ( $=R \ln 5 \approx 13.38 \mathrm{~J} \mathrm{~K}^{-1} \mathrm{~mol}^{-1}$ ), assuming that the RT phase is the disordered phase over $m$ conformations ignoring the disorder in the LT phase. The experimental magnitude $\left(\Delta S=7.59 \mathrm{~J} \mathrm{~K}^{-1} \mathrm{~mol}^{-1}\right)$ is certainly smaller than this maximum estimate. The butyl group is surely involved in the phase transition as evidenced by the disorder in the conformation of butyl groups in the crystal structure at room temperature and the appearances of distinct $\mathrm{CH}$-stretching bands in IR spectra at low temperatures (Fig. 4). If the twofold disorder of only a butyl group of the two attached to a BBCHM molecule detected by X-ray diffraction reflects the reality, the entropy increment involved would be $\frac{4}{5} R \ln 2\left(\approx 4.61 \mathrm{~J} \mathrm{~K}^{-1} \mathrm{~mol}^{-1}\right)$ because one molecule out of five independent molecules remains disordered at $100 \mathrm{~K}$. It is noted that the degree of disorder based on the crystallography for alkyl chains often gives a reasonable estimate of entropy even if it is against the intuition. ${ }^{22-25}$ However, this estimate is certainly smaller than the experimental finding. A possibility of another contribution is the disorder of $\mathrm{H}$-bonds in a cyclic tetramer. To fulfill the rule that only a single proton can be close to each oxygen atom (the "ice rule"), possible states for a cyclic tetramer concerning the sense of $\mathrm{H}$-bonds are either clockwise and counterclockwise. The expected contribution of this disorder is $\frac{1}{4} R \ln 2$ $\left(\approx 1.44 \mathrm{~J} \mathrm{~K}^{-1} \mathrm{~mol}^{-1}\right)$. Unfortunately, their sum is still definitely smaller than the experimental magnitude. It is interesting to see that the experimental entropy increment is comparable to $\frac{4}{5} R \ln 3\left(\approx 7.31 \mathrm{~J} \mathrm{~K}^{-1} \mathrm{~mol}^{-1}\right)$, which corresponds to the assumption that the disorder be not twofold but threefold for one butyl group of the two attached to a BBCHM molecule.

\section{CONCLUSION}

In this study, the experimental characterization of solid BBCHM is performed utilizing FT-IR, adiabatic calorimetry, and X-ray crystallography.

The FT-IR indicates the complete formation of an H-bond in a just enough way in both RT and LT phases, and its temperature-dependent dissociation in the liquid. In apparent contradiction with the complete formation of $\mathrm{H}$-bonds implied by the FT-IR result, the crystal structure at room temperature has a small unit cell with the disorder concerning the direction of the $\mathrm{C}-\mathrm{OH}$ bonds. This seeming disorder cannot be regarded as being dynamical in nature because of the bulkiness of a molecule. Suggested is a model that guarantees almost complete formation of $\mathrm{H}$-bonds and represents the disordered structure in average: A molecular pair and another in the adjacent cell (shifted by $\boldsymbol{b}$ ) form a tetramer with cyclic H-bonds. The stack along the $\boldsymbol{b}$-axis has a periodicity $2 \boldsymbol{b}$ and is nearly perfect, but their relative phases are random. This randomness recovers the higher symmetry than that of the crystal with the complete order.

The LT phase below $151.6 \mathrm{~K}$ has a large superstructure $(m=5)$ with respect to the RT phase. The character is discussed of the phase transition of BBCHM that a single modulation wave is sufficient to drive this cell-quintupling transition. Through a general consideration of possible superstructures, it is pointed out that this $m=5$ transition is of the minimum $m$ with full coverage of the whole reciprocal plane in a nontrivial way. Although there seem to be no examples sharing this character in the literature, a search over rather complex compounds like BBCHM will put a new stage for the understanding of structural phase transitions in an organized way after Landau.

\section{SUPPLEMENTARY MATERIAL}

See supplementary material for details of the synthesis of $\mathrm{BBCHM}$ and the experimental conditions and crystallographic parameters.

\section{ACKNOWLEDGMENTS}

This work was supported in part by a Grant-inAid for Scientific Research on Innovative Area "Fluctuation \& Structure" (No. 26103504) from the Ministry of 
Education, Culture, Sports, Science, and Technology of Japan. Mr. Y. Arai is indebted for his assistance in sample preparation.

${ }^{1}$ R. Kumai, S. Horiuchi, Y. Okimoto, and Y. Tokura, J. Chem. Phys. 125, 084715 (2006).

${ }^{2}$ M. Amano, Y. Yamamura, M. Sumita, S. Yasuzuka, H. Kawaji, T. Atake, and K. Saito, J. Chem. Phys. 130, 034503 (2006).

${ }^{3}$ G. Bator, W. Sawka-Dobrowlska, L. Sobczyk, E. Grech, J. NowickaScheibe, A. Pawlukojć, J. Wuttke, J. Baran, and M. Owczarek, J. Chem. Phys. 135, 044509 (2011).

${ }^{4}$ L. D. Landau and E. M. Lifshitz, Statistical Physics, 3rd ed. (ButterworthHeinemann, Oxford, 1980).

${ }^{5}$ P. G. de Genne and J. Prost, The Physics of Liquid Crystals, 3rd ed. (Clarendon Press, Oxford, 1993).

${ }^{6} \mathrm{~J} .-\mathrm{C}$. Tolédano and P. Tolédano, The Landau Theory of Phase Transitions (World Scientific, Singapore, 1987).

${ }^{7}$ H. T. Stokes and D. M. Hatch, Isotropy Subgroups of the 230 Crystallographic Space Groups (World Scientific, Singapore, 1988).

${ }^{8}$ Incommensurate Phases in Dielectrics, Fundamental, edited by V. M. Agranovich and A. A. Maradudin (North-Holland Physics Publishing, Amsterdam, 1986).

${ }^{9}$ Y. Yamamura, Y. Suzuki, M. Sumita, and K. Saito, J. Phys. Chem. B 116, 3938-3943 (2012).

${ }^{10}$ P. Sgarabotto, F. Ugozzoli, S. Sorriso, and Z. Malarski, Acta Crystallogr., Sect. C: Cryst. Struct. Commun. 44, 671-673 (1988).

${ }^{11}$ Y. Suzuki, Y. Yamamura, M. Sumita, S. Yasuzuka, and K. Saito, J. Phys. Chem. B 113, 10077-10080 (2009).
${ }^{12}$ S. Wang, M. M. Kayser, H. Iwaki, and P. C. K. Lau, J. Mol. Catal. B: Enzym. 22, 211-218 (2003).

${ }^{13}$ Y. Yamamura, K. Saito, H. Saitoh, H. Matsuyama, K. Kikuchi, and I. Ikemoto, J. Phys. Chem. Solids 56, 107-115 (1995).

${ }^{14}$ SAINT version 8.34A 2013, Bruker AXS, Madison, WI, 2013.

${ }^{15}$ G. M. Sheldrick, "SHELXS-97," Acta Crystallogr., Sect. A: Found. Crystallogr. 64, 112 (2008).

${ }^{16}$ G. M. Sheldrick, "SHELXL-2014,” Acta Crystallogr., Sect. C: Struct. Chem. 71, 3 (2015).

${ }^{17}$ Deposition numbers CCDC-1523471 (RT phase) and 1523473 (LT phase), copies of the data can be obtained free of charge via http//www.ccdc.cam.ac.uk/conts/retrieving.html, or from the Cambridge Crystallographic Data Centre, 12, Union Road, Cambridge, CB2 1EZ, UK, Fax: +44 1223 336033, e-mail: deposit@ccdc.cam.ac.uk.

${ }^{18}$ Y. Maréchal, The Hydrogen Bond and the Water Molecule (Elsevier, 2007).

${ }^{19}$ A. I. Kitaigorodsky, Molecular Crystals and Molecules (Academic Press, New York, 1973).

${ }^{20}$ CSD Space Group tatistics, the Statistics of the Cambridge Structural Database, Cambridge Crystallographic Data Centre, November 6, 2015, available at http://ccdc.cam.ac.uk.

${ }^{21}$ C. P. Brock, Acta Crystallogr., Sect. B: Struct. Sci., Cryst. Eng. Mater. 72, 807-821 (2016).

${ }^{22}$ S. Ikeuchi, K. Saito, Y. Nakazawa, A. Sato, M. Mitsumi, K. Toriumi, and M. Sorai, Phys. Rev. B 66, 115110 (2002).

${ }^{23}$ S. Ikeuchi, K. Saito, Y. Nakazawa, M. Mitsumi, K. Toriumi, and M. Sorai, J. Phys. Chem. B 108, 387-392 (2004).

${ }^{24}$ S. Ikeuchi, Y. Yamamura, Y. Yoshida, M. Mitsumi, K. Toriumi, and K. Saito, Bull. Chem. Soc. Jpn. 83, 261-266 (2010).

${ }^{25}$ S. Ikeuchi and K. Saito, Curr. Inorg. Chem. 4, 74-84 (2014). 